\title{
Future Optimal Dosing Regimens for Thrombolysis in Acute Stroke
}

\section{Kiyoshi Kikuchi ${ }^{1,2,3,4 *}$, Motohiro Morioka², Yoshinaka Murai', Eiichiro Tanaka ${ }^{1}$}

${ }^{1}$ Division of Brain Science, Department of Physiology, Kurume University School of Medicine, 67 Asahi-machi, Kurume 830-0011, Japan ${ }^{2}$ Department of Neurosurgery, Kurume University School of Medicine, 67 Asahi- machi, Kurume 830-0011, Japan

${ }^{3}$ Department of Systems Biology in Thromboregulation, Kagoshima University Graduate School of Medical and Dental Sciences, 8-35-1 Sakuragaoka, Kagoshima 8908520, Japan

${ }^{4}$ Department of Pharmacology, Faculty of Dentistry, Mahidol University, 6 Yothe Road, Rajthevee, Bangkok 10400, Thailand

\section{Short Commentary}

In 2012, stroke was the second most common cause of death worldwide [1]. One in 10 people with stroke die in hospital, and many more suffer ongoing disability, which highlights the need for more effective treatments [2].

Recently, the potential of endovascular therapy for acute ischemic stroke (AIS) using catheter-based devices was reported in five randomized clinical trials (RCTs). In all five RCTs that compared endovascular therapy after intravenous alteplase with intravenous alteplase alone, therapeutic superiority was shown in the former regimen [3-7]. However, standardization of endovascular therapy would exacerbate regional disparities owing due to the lack of endovascular specialists.

Efficacy of intravenous thrombolysis using alteplase $(0.9 \mathrm{mg} / \mathrm{kg}$ body weight), a recombinant tissue plasminogen activator, has been well established in Western countries since 1996 [8]. Further breakthroughs are needed for thrombolysis in acute stroke because intracerebral hemorrhage (ICH) after alteplase administration and a low prevalence of recanalization of occlusions of a major cerebral artery (e.g., internal carotid, proximal middle cerebral) limit the efficacy of alteplase [9].

In a study that compared $0.6,0.7,0.8$, and $0.9 \mathrm{mg} / \mathrm{kg}$ of alteplase in 1,004 eligible patients, Chao et al. reported that $0.9 \mathrm{mg} / \mathrm{kg}$ alteplase was not optimal for all patients in an East Asian population [10]. In that study, in elderly patients (71-80 years), mortality and symptomatic intracerebral hemorrhage $(\mathrm{SICH})$ increased with increasing alteplase dose, and a lower dose $(0.6 \mathrm{mg} / \mathrm{kg})$ of alteplase was associated with a better outcome [10]. Therefore, the optimal dose of alteplase may vary according to ethnicity and age. Currently, the Enhanced Control of Hypertension and Thrombolysis Stroke Study (ENCHANTED) is assessing the safety/efficacy of two doses of alteplase $(0.6,0.9 \mathrm{mg} / \mathrm{kg})$ in Australia, Brazil, Chile, China, Colombia, Hong Kong, Italy, Korea, Norway, Singapore, Taiwan, Thailand, the United Kingdom, and Vietnam [11]. ENCHANTED will reveal the optimal dose of alteplase according to ethnicity and age.

In Japan, the efficacy of alteplase $(0.6 \mathrm{mg} / \mathrm{kg})$ has been well established since 2005 [9]. The internationally recommended dose of alteplase is $0.9 \mathrm{mg} / \mathrm{kg}$. However, only $0.6 \mathrm{mg} / \mathrm{kg}$ is used in Japan, which could result in reduced efficacy [9]. Alteplase-edaravone combination therapy (AECT) has been shown to improve early outcomes in AIS patients compared with those treated with alteplase alone: in 356 pairs in a propensity-matched population, ordinal logistic regression analyses showed that addition of edaravone to alteplase therapy was associated significantly with lower Modified Rankin Scale scores upon hospital discharge [12].

As well as direct evidence from RCTs, circumstantial evidence suggests that edaravone has beneficial effects in AIS. Two large postmarketing surveys have been conducted: the Safe Implementation of Thrombolysis in Stroke-Monitoring Study (SITS-MOST) and the
Japan post-Marketing Alteplase Registration Study (J-MARS). In SITSMOST, the $0.9 \mathrm{mg} / \mathrm{kg}$ dose was evaluated in 6,483 European patients with AIS [13], whereas in J-MARS the $0.6 \mathrm{mg} / \mathrm{kg}$ dose was evaluated in 7,492 Japanese patients with AIS [14]. Prevalence of complete recovery at 3 months was identical in the two studies (39\%). Interestingly, in J-MARS, edaravone was also administered to $74.6 \%$ of 7,492 patients [15], suggesting that combination with edaravone enhanced the effectiveness of the reduced dose of alteplase used in Japanese patients with AIS.

Since its approval for use in Japan in 2001, edaravone (3-methyl1-phenyl-2- pyrazolin-5-one, Radicut ${ }^{\circledR}$, Mitsubishi Tanabe Pharma Corporation, Osaka, Japan) has entered routine clinical practice [9]. A relatively substantial body of clinical and experimental research has demonstrated that edaravone is a low-specificity antioxidant that scavenges singlet oxygen molecules, the superoxide anion as well as hydroxyl, alkoxyl, alkylperoxyl and methyl free radicals, and exhibits neurovascular protective effects against apoptosis, necrosis, edema and inflammatory cytokines [16-27]. Edaravone is a low-molecular-weight agent with water- and lipid-soluble properties $[28,29]$. Unlike other free radical-scavengers, edaravone crosses the blood-brain barrier readily, which may explain its therapeutic benefits $[28,29]$. Furthermore, AECT has been shown to reduce infarct size in a comparison between AECT and alteplase alone in preclinical models $[26,27,30]$. The fibrin-binding affinity of alteplase can be impaired by exposure to reactive oxygen species (ROS), and the characteristic advantage of the thrombus selectivity of alteplase in spontaneous thrombolysis and thrombolytic therapy may be diminished in environments in which ROS are plentiful [31]. Moreover, edaravone appears to ameliorate alteplase-induced oxidative stress in rat brains [24].

Edaravone is not approved for use in Western countries. The treatment protocol in Japan comprises a twice-daily intravenous infusion administered for $\leq 14$ days. Edaravone has a very short halflife $\left(T_{1 / 2}=5.4 \mathrm{~min}\right)[32]$, and free radicals are generated soon after vessel occlusion and reperfusion [33]. Biomarkers of oxidative stress are already raised before recanalization in patients with AIS treated subsequently with alteplase [34]. Recently, Kaste et al. reported that a

*Corresponding author: Kiyoshi Kikuchi, Division of Brain Science, Department of Physiology, Kurume University School of Medicine, 67 Asahimachi, Kurume 830-0011, Japan, Tel: +81-942-31-7542; Fax: +81-942-31-7695; E-mail:kikuchi_kiyoshi@kurume-u.ac.jp

Received: January 23, 2016; Accepted: January 27, 2016; Published February 01,2016

Citation: Kikuchi K, Tancharoen S, Murai Y, Tanaka E (2016) Future Optimal Dosing Regimens for Thrombolysis in Acute Stroke. Biochem Anal Biochem 5 : 244. doi:10.4172/2161-1009.1000244

Copyright: $\odot 2016$ Kikuchi K, et al. This is an open-access article distributed unde the terms of the Creative Commons Attribution License, which permits unrestricted use, distribution, and reproduction in any medium, provided the original author and source are credited. 
new formulation and dosing regimen of edaravone (high-dose, 3-day continuous infusion) was safe for patients with AIS [35]. It will be interesting to see if that study, conducted in Finland, the Netherlands and the United Kingdom, will influence or accelerate use of edaravone in the European Union.

In a comparison between AECT and alteplase alone in preclinical models, the former ameliorated thrombolysis-related hemorrhage $[26,27]$. Edaravone attenuated alteplase-mediated matrix metalloproteinase-9 (MMP-9) production, which in turn protected against blood-brain barrier breakdown [26,27]. As well as its effects in preclinical studies, circumstantial evidence suggests that edaravone has beneficial effects in AIS that may be explained by a reduction in the prevalence of adverse events caused by alteplase. Prevalence of SICH development attributed to alteplase infusion is reportedly negatively correlated with the prevalence of combined treatment with edaravone in several RCTs. None of the 6,483 participants in SITS-MOST or the 103 participants in the Japan Alteplase Clinical Trial (J-ACT) received edaravone. However, most participants received edaravone in J-MARS and J-ACT II trials (5,557 of 7,492 participants (74.2\%), and 53 of 58 participants (91.4\%), respectively) [8]. Prevalence of symptomatic ICH was $8.5 \%$ in SITS-MOST, $5.8 \%$ in J-ACT, 3.5\% in J-MARS, and $0.0 \%$ in J-ACT II [8]. Those results suggest that edaravone may inhibit hemorrhagic transformation in patients with AIS who receive thrombolysis with alteplase.

RCTs focusing on dose modification of alteplase, next-generation agents of t-PA (tenecteplase, desmoteplase), endovascular therapy, and sonothrombolysis are ongoing worldwide [9], but AECT should be highlighted. Synergistic effects of AECT are accepted widely, and almost all Japanese patients with AIS receive edaravone. Therefore, it may be difficult for large RCTs that compare alteplase plus edaravone with alteplase alone to be carried out in Japan owing to ethical reasons. AECT requires further investigation in large RCTs in Western countries to ascertain its efficacy unequivocally. Alteplase $0.9 \mathrm{mg}$ / $\mathrm{kg}$ plus edaravone (high-dose, 3-day continuous infusion) must be compared with alteplase $0.9 \mathrm{mg} / \mathrm{kg}$ in RCTs to show whether such a combination is a breakthrough treatment for AIS. The US Food and Drug Administration has granted edaravone an orphan drug designation for treatment of amyotrophic lateral sclerosis (ALS) [36]. This took place after edaravone was approved as having additional indication for ALS in Japan in 2015 [37]. Therefore, study of AECT in Western countries might also be carried out in the near future. We should remain optimistic about the future of therapeutic intervention for stroke, and continue to explore new strategies to provide optimal care for patients with AIS.

\section{Acknowledgments}

This review was supported by grants from the General Insurance Association of Japan. We thank Yoko Tsurusaki, Sachiko Nakashima, Rumi Ito and Tomoko Matsuo for their invaluable assistance.

\section{References}

1. http://www.who.int/mediacentre/factsheets/fs310/en/.

2. http://www.enchanted.org.au/.

3. Berkhemer OA, Fransen PS, Beumer D, van den Berg LA, Lingsma HF, et al (2015) A randomized trial of intraarterial treatment for acute ischemic stroke. N Engl J Med 372: 11-20.

4. Campbell BC, Mitchell PJ, Kleinig TJ, Dewey HM, Churilov L, et al. (2015) Endovascular therapy for ischemic stroke with perfusion-imaging selection. $\mathrm{N}$ Engl J Med 372: 1009-1018.

5. Goyal M, Demchuk AM, Menon BK, Eesa M, Rempel JL, et al. (2015) Randomized assessment of rapid endovascular treatment of ischemic stroke.
N Engl J Med 372: 1019-1030.

6. Khatri P, Yeatts SD, Mazighi M, Broderick JP, Liebeskind DS, et al. (2014) Time to angiographic reperfusion and clinical outcome after acute ischaemic stroke: An analysis of data from the interventional management of stroke (IMS III) phase 3 trial. Lancet Neurol 13: 567-574

7. Saver JL, Goyal M, Bonafe A, Diener HC, Levy El, Pereira VM, et al. (2015) Stent- retriever thrombectomy after intravenous t-PA vs. t-PA alone in stroke. N Engl J Med 372: 2285-2295.

8. Kikuchi K, Miura N, Kawahara KI, Murai Y, Morioka M, et al. (2013) Edaravone (radicut), a free radical scavenger, is a potentially useful addition to thrombolytic therapy in patients with acute ischemic stroke (review). Biom Rep 1: 7-12.

9. Kikuchi K, Tanaka E, Murai Y, Tancharoen S (2014) Clinical trials in acute ischemic stroke. CNS Drugs 28: 929-938.

10. Chao AC, Liu CK, Chen CH, Lin HJ, Liu CH, et al. (2014) Different doses of recombinant tissue-type plasminogen activator for acute stroke in chinese patients. Stroke 45: 2359-2365.

11. Anderson CS, Woodward M, Arima H, Chen X, Lindley RI, et al. (2015) Statistica analysis plan for evaluating low- vs. standard-dose alteplase in the ENhanced Control of Hypertension and Thrombolysis strokE stuDy (ENCHANTED) Int $\mathrm{J}$ Stroke 10: 1313-1315.

12. Wada T, Yasunaga H, Inokuchi R, Horiguchi H, Fushimi K, et al. (2014) Effects of edaravone on early outcomes in acute ischemic stroke patients treated with recombinant tissue plasminogen activator. J Neurol Sci 345: 106-111.

13. Wahlgren N, Ahmed N, Davalos A, Ford GA, Grond M, et al. (2007) Thrombolysis with alteplase for acute ischaemic stroke in the Safe Implementation of Thrombolysis in Stroke-Monitoring Study (SITS-MOST): an observational study. Lancet 369: 275-282.

14. Nakagawara J, Minematsu K, Okada Y, Tanahashi N, Nagahiro S, et al. (2010) Thrombolysis with $0.6 \mathrm{mg} / \mathrm{kg}$ intravenous alteplase for acute ischemic stroke in routine clinical practice: the Japan post-Marketing Alteplase Registration Study (J-MARS). Stroke 41: 1984-1989.

15. Kimura K, Aoki J, Sakamoto Y, Kobayashi K, Sakai K, et al. (2012) Administration of edaravone, a free radical scavenger, during t-pa infusion can enhance early recanalization in acute stroke patients--a preliminary study. J Neurol Sci 313: 132-136.

16. Kamogawa E, Sueishi Y (2014) A multiple free-radical scavenging (MULTIS) study on the antioxidant capacity of a neuroprotective drug, edaravone as compared with uric acid, glutathione, and trolox. Bioorg Med Chem Lett 24 1376-1379.

17. Kikuchi K, Kawahara K, Miyagi N, Uchikado H, Kuramoto T, et al. (2010) Edaravone: A new therapeutic approach for the treatment of acute stroke. Med Hypotheses 75: 583-585.

18. Kikuchi K, Kawahara K, Tancharoen S, Matsuda F, Morimoto Y, et al. (2009) The free radical scavenger edaravone rescues rats from cerebral infarction by attenuating the release of high-mobility group box-1 in neuronal cells. $J$ Pharmacol Exp Ther 329: 865-874.

19. Kikuchi K, Kawahara KI, Uchikado H, Miyagi N, Kuramoto T, et al. (2011) Potential of edaravone for neuroprotection in neurologic diseases that do not involve cerebral infarction. Exp Ther Med 2: 771-775

20. Kikuchi K, Takeshige N, Miura N, Morimoto Y, Ito T, et al. (2012) Beyond free radical scavenging: Beneficial effects of edaravone (radicut) in various diseases (review). Exp Ther Med 3: 3-8.

21. Kikuchi K, Tancharoen S, Matsuda F, Biswas KK, Ito T, et al. (2009) Edaravone attenuates cerebral ischemic injury by suppressing aquaporin-4. Biochem Biophys Res Commun 390: 1121-1125.

22. Kikuchi K, Tancharoen S, Takeshige N, Yoshitomi M, Morioka M, et al. (2013) The efficacy of edaravone (radicut), a free radical scavenger, for cardiovascular disease. Int J Mol Sci 14: 13909-13930.

23. Kikuchi K, Uchikado $\mathrm{H}$, Miyagi N, Morimoto $\mathrm{Y}$, Ito $\mathrm{T}$, et al. (2011) Beyond neurological disease: New targets for edaravone (review). Int J Mol Sci 28 899-906.

24. Lukic-Panin V, Deguchi K, Yamashita T, Shang J, Zhang X, et al. (2010) Free radical scavenger edaravone administration protects against tissue plasminogen activator induced oxidative stress and blood brain barrier damage. Curr Neurovasc Res 7: 319-329. 
Citation: Kikuchi K, Tancharoen S, Murai Y, Tanaka E (2016) Future Optimal Dosing Regimens for Thrombolysis in Acute Stroke. Biochem Anal Biochem 5: 244. doi:10.4172/2161-1009.1000244

Page 3 of 3

25. Tanaka E, Niiyama S, Sato S, Yamada A, Higashi H. (2003) Arachidonic acid metabolites contribute to the irreversible depolarization induced by in vitro ischemia. J Neurophysiol 90: 3213-3223.

26. Yagi K, Kitazato KT, Uno M, Tada Y, Kinouchi T, et al. (2009) Edaravone, a free radical scavenger, inhibits MMP-9-related brain hemorrhage in rats treated with tissue plasminogen activator. Stroke 40: 626-631.

27. Yamashita T, Kamiya T, Deguchi K, Inaba T, Zhang H, et al. (2009) Dissociation and protection of the neurovascular unit after thrombolysis and reperfusion in ischemic rat brain. J Cereb Blood Flow Metab 29: 715-725.

28. Watanabe T, Tahara M, Todo S (2008) The novel antioxidant edaravone: From bench to bedside. Cardiovasc Ther 26: 101-114.

29. Yoshida H, Yanai H, Namiki Y, Fukatsu-Sasaki K, Furutani N, et al. (2006) Neuroprotective effects of edaravone: A novel free radical scavenger in cerebrovascular injury. CNS Drug Rev 12: 9-20.

30. Sun YY, Morozov YM, Yang D, Li Y, Dunn RS, et al. (2014) Synergy of combined tPA-edaravone therapy in experimental thrombotic stroke. PLoS One 9: 98807.

31. Feng YH, Hart G (1995) In vitro oxidative damage to tissue-type plasminogen activator: A selective modification of the biological functions. Cardiovasc Res 30: 255-261.

32. Watanabe T, Yuki S, Egawa M, Nishi H. (1994) Protective effects of MCl-186 on cerebral ischemia: Possible involvement of free radical scavenging and antioxidant actions. J Pharmacol Exp Ther 268: 1597-1604.

33. Zhang W, Sato K, Hayashi T, Omori N, Nagano I, et al. (2004) Extension of ischemic therapeutic time window by a free radical scavenger, edaravone, reperfused with tpa in rat brain. Neurol Res 26: 342-348.

34. Dominguez C, Delgado P, Vilches A, Martin-Gallan P, Ribo M, et al. (2010) Oxidative stress after thrombolysis-induced reperfusion in human stroke. Stroke 41: 653-660.

35. Kaste M, Murayama S, Ford GA, Dippel DW, Walters MR, et al. (2013) Safety, tolerability and pharmacokinetics of $\mathrm{MCl}-186$ in patients with acute ischemic stroke: New formulation and dosing regimen. Cerebrovasc Dis 36: 196-204.

36. FDA Grants Edaravone Orphan Drug Designation for Treatment of Amyotrophic Lateral Sclerosis

37. http://www.alstdi.org/news/radicut-approved-for-als-in-japan/. 